\title{
Leveraging a temperature-tunable, scale-like microstructure to produce multimodal, supersensitive sensors $\uparrow$
}

\author{
Yanlong Tai, (D) a,b Tushar Kanti Bera, ${ }^{a}$ Zhenguo Yang ${ }^{\star b}$ and Gilles Lubineau*a
}

The microstructure of a flexible film plays an important role in its sensing capability. Here, we fabricate a temperature-dependent wrinkled single-walled carbon nanotube (SWCNT)/polydimethyl-siloxane (PDMS) film (WSPF) and a wrinkle-dependent scale-like SWCNT/PDMS film (SSPF) successfully, and address the formation and evolution mechanisms of each film. The low elastic modulus and high coefficient of thermal expansion of the PDMS layer combined with the excellent piezoresistive behavior of the SWCNT film motivated us to investigate how the scale-like microstructure of the SSPF could be used to design multimodal-sensing devices with outstanding capabilities. The results show that SSPFs present supersensitive performance in mechanical loading (an effective sensitivity of up to $740.7 \mathrm{kPa}^{-1}$ ) and in temperature (a tunable thermal index of up to $29.9 \times 10^{3} \mathrm{~K}$ ). These exceptional properties were demonstrated in practical applications in a programmable flexile pressure sensor, thermal/light monitor or switch, etc., and were further explained through the macroscopic and microscopic piezoresistive behaviors of scale-like SWCNT coatings.

Received 8th March 2017, Accepted 3rd May 2017

DOI: $10.1039 / \mathrm{c} 7 \mathrm{nr} 01662 \mathrm{j}$ rsc.li/nanoscale

\section{Introduction}

Mechanical buckling is a very common physical phenomenon in laminated structures ${ }^{1-3}$ that is often considered to be detrimental because it can initiate delamination between the layers. ${ }^{4,5}$ Yet, because buckling is simple, fast, and easy to trigger, without the use of complex or expensive instruments, it is also considered to be beneficial because it is an excellent approach to fabricate uniform patterns in thin coatings $\mathrm{s}^{6,7}$ with application in flexible electronics, ${ }^{8}$ optical designs, ${ }^{9}$ microfluidics, ${ }^{10}$ metrology, ${ }^{11}$ and biological assays. ${ }^{12}$ Investigation into the buckling mechanism of coatings ${ }^{13,14}$ has revealed that buckling is generated through residual stress at the interface between adjacent layers. The wavelength and amplitude of the pattern after buckling can be calculated or controlled via empirical equations. ${ }^{15-17}$

Sensors made with a nanomaterial (e.g., silver nanowire, graphene, carbon nanotube) component and a polydimethyl-

${ }^{a}$ King Abdullah University of Science and Technology (KAUST), Physical Sciences and Engineering Division, COHMAS Laboratory, Thuwal 23955-6900, Saudi Arabia. E-mail: gilles.lubineau@kaust.edu.sa

${ }^{b}$ Fudan University, Department of Materials Science, Shanghai 200433, China.

E-mail: zgyang@fudan.edu.cn

$\dagger$ Electronic supplementary information (ESI) available. See DOI: 10.1039/ c7nr01662j siloxane (PDMS) substrate have bilayered films that can be advantageous for various sensing applications. ${ }^{18-20}$ These films are ideal for sensing pressure/strain or temperature because they exploit the properties of both components: the low stiffness (Young's modulus around $2 \mathrm{MPa})^{21}$ and high temperature sensitivity (coefficient of thermal expansion (CTE), $\alpha=310 \times 10^{-6} \mathrm{~K}^{-1}$ ) of the PDMS substrate ${ }^{22,23}$ and the outstanding piezoresistive behavior of the nanomaterial component. ${ }^{24,25}$ The difference in stiffness and thermal expansion between the two layers can cause compressive residual stresses that are generated during the deposition process of the functional layer (here the nanoparticle-based layer), resulting in buckling patterns. ${ }^{26,27}$ Larger residual stresses can be obtained by prestraining the PDMS film during fabrication, amplifying the buckling process. ${ }^{28,29}$

Here, we take advantage of this buckling, and we demonstrate how buckling-induced patterning can be used to realize a supersensitive, programmable multimodal-sensing device.

First, we fabricate a temperature-dependent wrinkled single-walled carbon nanotube (SWCNT)/PDMS film (WSPF) and then through rubbing the film, we develop a wrinkledependent scale-like SWCNT/PDMS film (SSPF). We present how well-known theoretical aspects of mechanical buckling guided our design of each film to achieve specific characteristics. We also investigate the relationships between the microstructure and electrical resistance of each film. 
Second, we investigate the mechanical-pressure/ temperature-/light-sensing capabilities (monitoring and switching) of SSPFs to confirm their supersensitive performances. We perform a series of practical applications, including accurately monitoring the indicators of human health, weighing an insect (ant) and tracking its activities, gauging temperature, designing a thermal switch in a fire alarm system, as well as monitoring light.

Third, we explain the excellent sensing performance of SSPF films based on their novel scale-like microstructure: (1) changing the deformation from 3-dimensional (3D) to 2D, and totally transferring the deformation of the film to resistance variation of the CNT layer $\left(\mathrm{cm} \mathrm{mm}^{-1}\right.$ scale); (2) two kinds of piezoresistive behaviors (micro/nanoscale): the variation in the distance between the scale-like SWCNT islands (SSIs) and the deformation of SSIs. Both mechanisms work in synergy to promote the macroscopic properties of the sensors.

\section{Results and discussion}

\section{Preparation of WSPFs and SSPFs}

As for WSPFs, when a material with a higher Young's modulus is deposited as a thin coating onto a pre-heated PDMS film, a homogeneous wave-like microstructure forms through buckling during cooling. Buckling theory ${ }^{13,16}$ explains the buckling behavior of these film-on-elastomer structures as being related to the residual stress, the materials, and the geometric parameters of the film. The strength of the interfacial adhesion between the substrate and the coating is also important as buckling may induce delamination between the two layers. The emergence of the buckled microstructure is the result of the equi-biaxial compressive stress that originates because of the differences in CTEs between the two layers. Note that PDMS has a high CTE value at $310 \times 10^{-6} \mathrm{~K}^{-1}$, indicating its strong thermal prestrain capability, whereas SWCNTs have a slightly negative CTE value $\left(1.5 \times 10^{-6} \mathrm{~K}^{-1}\right)$. The compressive stress in the coating before buckling $\left(\sigma_{0}\right)$ can be calculated by eqn (1), ${ }^{13}$ along with wavelength $(\lambda)$ and amplitude $(A)$, as given from eqn (2). ${ }^{17}$

$$
\sigma_{0}=\frac{E_{\mathrm{f}}\left(\alpha_{\mathrm{S}}-\alpha_{\mathrm{f}}\right)\left(T_{2}-T_{1}\right)}{1-\nu_{\mathrm{f}}}
$$

$$
\lambda=2 \Pi h\left(\frac{E_{\mathrm{f}}}{3 E_{\mathrm{s}}}\right)^{1 / 3}, \quad A=h \sqrt{\frac{\varepsilon_{\mathrm{pre}}}{\varepsilon_{\mathrm{c}}}-1}\left(\varepsilon_{\mathrm{c}}=0.25\left(\frac{3 E_{\mathrm{s}}}{E_{\mathrm{f}}}\right)^{2 / 3}\right)
$$

where $E_{\mathrm{f}}$ and $\alpha_{\mathrm{f}}, E_{\mathrm{s}}$ and $\alpha_{\mathrm{s}}$ are the Young's modulus and CTE of the nanomaterial film (SWCNT) and the substrate (PDMS), respectively; $\nu_{\mathrm{f}}$ is the Poisson ratio of the film; $T_{2}$ and $T_{1}$ are the deposition temperature and final temperature, respectively; $\varepsilon_{\mathrm{c}}$ is the buckling critical strain; $\varepsilon_{\text {pre }}$ is the strain provided by thermal expansion; and $h$ is the thickness of the film.

Based on the preparation process in Fig. $1 \mathrm{a}$ and $\mathrm{b}$, the results in Fig. 1c, d, and $\mathrm{S} 1 \uparrow$ demonstrated that the microstructure of WSPFs changes dramatically as the drying temperature increases from $50{ }^{\circ} \mathrm{C}$ to $100^{\circ} \mathrm{C}$ to $150^{\circ} \mathrm{C}$. Meanwhile, the wave-
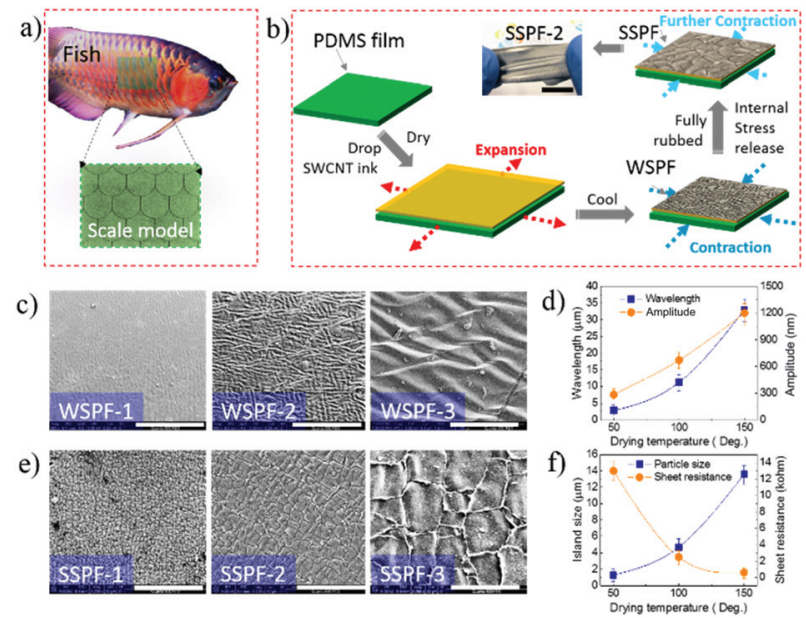

Fig. 1 Preparation and characterization of WSPFs and SSPFs with different microstructures. (a) Digital image of fish-scale model. (b) Illustration of the preparation process of scale-like microstructures. Scale bar is $1 \mathrm{~cm}$. (c) SEM images of WSPFs produced with different drying temperatures $\left(50^{\circ} \mathrm{C}\right.$ : WSPF- $1,100^{\circ} \mathrm{C}$ : WSPF-2, $150^{\circ} \mathrm{C}$ : WSPF-3); scale bar represents $100 \mu \mathrm{m}$. (d) Relationship between drying temperature, wavelength, and amplitude of the buckled patterns. (e) SEM images of SSPFs produced from the corresponding WSPFs in (c); scale bar represents $20 \mu \mathrm{m}$. (f) Relationship between drying temperature, SWCNT island size, and initial sheet resistance of WSPFs. The default thickness of PDMS was $200 \pm 10 \mu \mathrm{m}$.

length increases from $2.8 \mu \mathrm{m}$ to $11.2 \mu \mathrm{m}$ to $33 \mu \mathrm{m}$, and the amplitude increases from $287 \mathrm{~nm}$ to $670 \mathrm{~nm}$ to $1211 \mathrm{~nm}$. These results were expected from eqn (1) and (2). Indeed, when curing is performed at higher temperatures, the critical strain to buckling is also reached at higher temperatures during cooling. As the Young's modulus of the PDMS decreases with decreasing temperature, both the wavelength and the amplitude increase with the increased curing temperature.

As for SSPFs, by simply rubbing the samples with our hands, we caused the SWCNT layer to crack, producing a homogeneous microstructure, as shown in Fig. S1 and S2. $\dagger$ This microstructure was similar to the previous mechanical pattern obtained by buckling, as shown in Fig. 1e and f. The in-plane dimension of the scale-like islands was guided completely by the initial half-wavelength of the pattern after buckling: $1.3 \mu \mathrm{m}$ for SSPF-1 (half-wavelength after buckling: $1.4 \mu \mathrm{m}$ ), $4.7 \mu \mathrm{m}$ for SSPF-2 (half-wavelength after buckling: $5.6 \mu \mathrm{m}$ ), and $13.6 \mu \mathrm{m}$ for SSPF-3 (half-wavelength after buckling: $16.5 \mu \mathrm{m})$.

These cracks have a strong influence on the performance of these bilayered films. For example, the sheet resistance of films increases as the number of cracks increases from $0.6 \mathrm{kohm} \mathrm{sq}^{-1}$ to $2.3 \mathrm{kohm} \mathrm{sq}^{-1}$ to $13.2 \mathrm{kohm} \mathrm{sq}^{-1}$ compared with the original $116 \mathrm{ohm} \mathrm{sq}{ }^{-1}$, as shown in Fig. 1f. Each scalelike surface introduced an additional contact resistance in the film that results in a higher macroscopic resistance when crack density increases. In addition, recall that the original film (before cracking) was highly temperature sensitive, demon-
1 
strating large deformations due to the large difference in thermal strains between the layers. This behavior makes it a potentially good candidate for designing actuators. However, after cracking, the laminate no longer bent when subjected to temperature or light. Instead, it freely deformed in its plane due to cracks that had been introduced between the coating fragments. The difference in the ability of films to bend can be seen in Fig. S3. $\dagger$

\section{Sensing performance of SSPFs in mechanical pressure}

Wearable pressure sensors are often PDMS based due to the low Young's modulus of PDMS. A common strategy to improve the sensitivity of these sensors is to change the contact surface to a point of contact at the sensing interface. However, to maintain the stability, a preload has to be applied, which reduces the expected performance of the sensor. ${ }^{30,31}$ Here, SSPFs were integrated into a polyethylene terephthalate (PET) film with a silver pattern serving as the electrode and an array hole serving as the sensing unit, together forming a pressure-sensing system. More details can be found in the Experimental section.

Fig. 2a shows a positive relationship between the increased resistance of the pressure-sensing device and the increased loading values until the break. The relationship between $X$-log/ $Y$ - $\log$ values is nearly linear within the effective pressuresensing scope (SSPF-1: 40.5 kPa, SSPF-2: $56.7 \mathrm{kPa}$, SSPF-3: 72.9
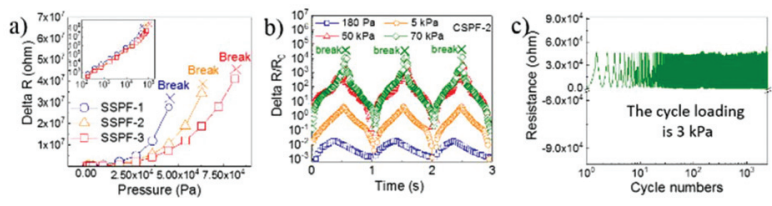

d)
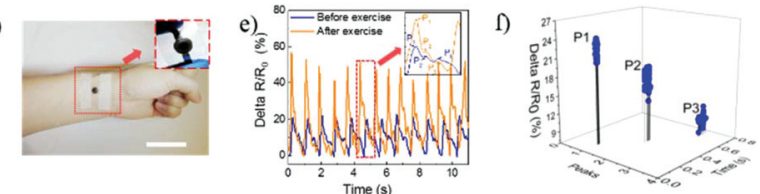
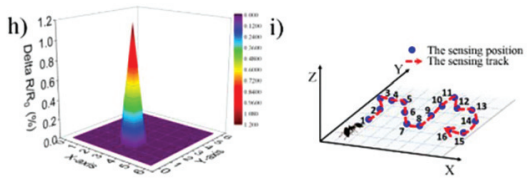

Fig. 2 Sensing performance of SSPFs in response to mechanical pressure. (a) Resistance variations of different SSPF-based pressuresensing devices as a function of mechanical pressure; inset illustrates an $X / Y$-log plot. (b) Multi-cycle tests of dynamic loading with different loading pressures. (c) Durability test under a cycle loading of $3 \mathrm{kPa}$ and frequency of $1 \mathrm{~Hz}$; (d) a digital image showing a wearable sensor directly attached to the artery of the tester's wrist; scale bar is $3 \mathrm{~cm}$. (e) Resistance-response measurements of a human heartbeat at rest (61 beats per $\mathrm{min}$ ) and following exercise (74 beats per min). (f) Statistical distribution of key data $\left(P_{1}, P_{2}\right.$, and $P_{3}$ with the relevant time intervals) from a 33-year-old man. (g) A digital image of a $6 \times 6$ pressure-sensing array $(9 \mathrm{~cm} \times 9 \mathrm{~cm})$ with pressure applied by an ant (red); scale bar is $2 \mathrm{~cm}$. (h) 3D map of calculated intensity and position of the ant and (i) the continuous sensing of the ant from positions no. 1 to no. 16. SSPF-2 was used as the default flexible film. All data points are the average of three samples. The default loading speed was $12 \mathrm{~mm} \mathrm{~min}^{-1}$.
$\mathrm{kPa}$ ). Note that the preferred effective scope is $90 \%$ of the breaking pressure load.

The response of the current sensing system differs from our results in previous works regarding the deformation of circle-clamped PET ${ }^{32,33}$ because the Young's modulus of PET $(2-2.7 \mathrm{GPa})$ is so high that its deformation becomes more difficult as loading values increase. Using PDMS, the influence of the Young's modulus can be neglected (around $2 \mathrm{MPa}$ ). Thus, when an external load is applied within the hole area on SSPF (PDMS side), a sharp variation in resistance occurs as a result of the scale-like microstructure. In previous work, we noted that, according to classic thin-plate theory, the deflection of the circle-clamped SSPF is strongly related to the diameter of the hole. ${ }^{30,34}$ In other words, the pressure-sensing capability of the film is adjustable. Thus, to focus on SSPFs themselves, here, we maintained a default PET circle diameter of $6 \mathrm{~mm}$. This SSPF has a large detecting scope, meaning that the variation in the resistance of these devices can change stably and continuously from several kohm to tens of Mohm before break. Furthermore, calculated film sensitivities $(S$, is defined as delta $R / R_{0}$ to pressure) exceeded our expectations: SSPF-1: $36.5 \mathrm{kPa}^{-1}$, SSPF-2: $216.9 \mathrm{kPa}^{-1}$, and SSPF-3: 740.7 $\mathrm{kPa}^{-1}$ compared with $192 \mathrm{kPa}^{-1}$ reported previously as the best value in the working range of $5 \mathrm{kPa}$ (refer to Table S1 and Fig. S4†).

Fig. $2 \mathrm{~b}$ shows the cycling performance of the film at loading values between $180 \mathrm{~Pa}$ (the lowest value that could be applied to our instrument) and $70 \mathrm{kPa}$ (resistance break). All curves show a sharp signal with low noise, satisfying repeatability and indicating that the interfacial layers of the laminated film remained stable, which is particularly important for our scale-like structure after the rubbing process.

Fig. 2c illustrates the durability of the film where a single sensing unit maintains a clear signal after 2000 cycles with negligible changes in the amplitude of its resistance. This result not only confirms the efficiency of the packaging technology via the transferred nano-sized PDMS film and plasma treatment, but also the strong adhesive performance between the scale-like SWCNT layer and the PDMS layer.

Next, we illustrated the efficiency of our pressure-sensing system by demonstrating its ability to monitor continuous wrist pulse signals, as shown in Fig. $2 \mathrm{~d}$ and Video S1. $\dagger$ The results in Fig. 2e show accurate readings of wrist pulse at rest ( 66 beats per min) and after physical exercise ( 75 beats per min). In addition, we made a 1 min continuous pulse recording from a 33-year-old man, and we removed the key data ( $P 1$, $P 2$, and $P 3$ with the relevant time intervals) to plot them in a 3-dimensional image (Fig. 2f). The results verified the potential of this sensing system as a wearable diagnostic device for monitoring human health in real-time.

We further confirmed the efficiency of the device by calculating the intensity of the variation in resistance (variation in weight of the ant) and continuously mapping the movement of an ant through recording the variation in resistance at each sensing unit in the $X-Y$ plane from positions no. 1 to no. 16 , as shown in Fig. $2 \mathrm{~g}-\mathrm{i}^{35}$ 


\section{Thermal sensing of SSPFs}

From previous reports, we know that the carbon nanotube/ PDMS laminate has a strong thermal/light-deforming capability and can even bend due to the mismatch in coefficients of thermal expansion (CTE, $\alpha_{\text {SWCNT }}=-1.5 \times 10^{-6} \mathrm{~K}^{-1}, \alpha_{\mathrm{PDMS}}=$ $\left.310 \times 10^{-6} \mathrm{~K}^{-1}\right) \cdot{ }^{36,37}$ However, after being fully rubbed, more
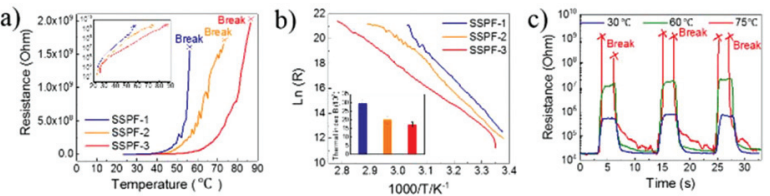

d)
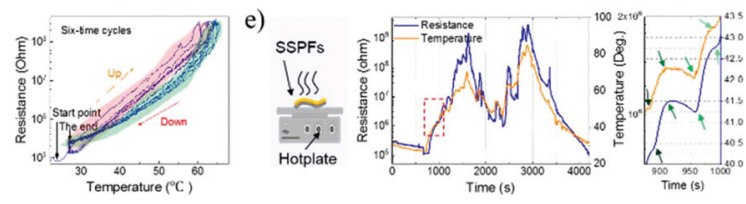

f)
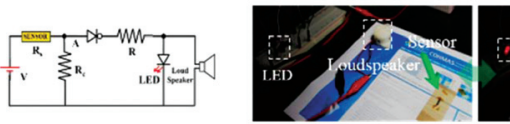

Fig. 3 Applications of SSPFs to thermal sensing. (a) Variation in resistance of each type of $1 \mathrm{~cm} \times 2 \mathrm{~cm} \mathrm{SSPF}$ as a function of temperature; inset is the $X-\log (Y)$ plots. (b) Linear dependence of $\ln (R)$ on $1000 / T$; inset is the thermal index B calculated from the thermal index value of different SSPFs. (c) Variation in resistance with temperature of SSPF-2. (d) The relationship between resistance and temperature over six time cycles. (e) Thermal monitoring test via resistance response. The graph on the right is a magnification of the red rectangle highlighted on the main plot. (f) The configuration and images of the temperature alarm system (green arrows) point to $2 \mathrm{~mm} \times 1 \mathrm{~cm}$ SSPFs. All data points are the average of three samples. observed for typical transition metal oxides (around 3.5-15 times). For more details, refer to Table S2 and Fig. S5. $\dagger^{39,40}$

$$
\ln (R)=\ln \left(R_{0}\right)+\frac{B}{T}
$$

where $R$ is the resistance at temperature $T$, and $R_{0}$ is the resistance at $T=\infty$.

Fig. 3c shows the multi-cycle tests of SSPF-2 at different temperatures $\left(30^{\circ} \mathrm{C}, 60^{\circ} \mathrm{C}\right.$, and $\left.75^{\circ} \mathrm{C}\right)$. Such regular and continuous response profiles can be obtained thanks to the outstanding cyclic properties of PDMS. In addition, the excellent thermal cycle is also presented in Fig. $3 \mathrm{~d}$.

The efficiency of these SSPFs to monitor temperature was investigated in Fig. 3e. The results show that this film can detect variation in temperature accurately within $90 \%$ of the scope of its breaking temperature. In addition, between $35^{\circ} \mathrm{C}-$ $45{ }^{\circ} \mathrm{C}$, SSPF-2 can sense changes in temperature as small as $0.05{ }^{\circ} \mathrm{C}$. This excellent performance indicates its promising application as a thermometer.

Although these SSPFs are efficient within the scope of their working temperatures, once the temperature surpasses the breaking temperature, the circuit immediately breaks. For this reason, these SSPFs also have the potential to act as a thermal switch. Fig. 3f illustrates how this film might be applied to a fire alarm system, and more details are explained in the Experimental section. We found that when a hot stick (around $250{ }^{\circ} \mathrm{C}$ ) came in close enough proximity to SSPF-2, the LED began to shine, causing a loudspeaker to sound an alarm, as shown in Video S3. $\dagger$ We also investigated the light-monitoring and switching performance of SSPFs, based on the mechanism of thermal deformation described above, as shown in Fig. S6 and Video S4.†

\section{Analysis of the sensing mechanism of SSPFs}

To further understand the sensing mechanism of SSPFs, the change in the impedance of SSPF-2 was monitored via electrochemical impedance spectroscopy (EIS) at different temperatures, as shown in Fig. 4a. As expected, both the real and complex parts of the impedance increase with increasing temperature due to modifications in the morphology of the conductive network. When temperature is increased, due to its discontinuous SWCNT layer, SSPF undergoes only the 2D deformation, not $3 \mathrm{D}$, though it is an actuating material. This phenomenon is very important to transfer the deformation of film to resistance variation of the CNT layer $\left(\mathrm{cm} \mathrm{mm}^{-1}\right.$ scale) totally. Meanwhile, two main modifications are expected in the morphology of the scale-like coating: (1) the opening of the cracks between the fragments, resulting in an increase in impedance in between the fragments and (2) stretching of the fragments, resulting in an increase in intrinsic impedance of the fragments.

To highlight each of these piezo mechanisms, the change in the microstructure of SSPF-2 under different uniaxial strains was characterized, as shown in Fig. S7.† Both the opening of the cracks in between the fragments and the stretching of the fragments are clearly visible here. 

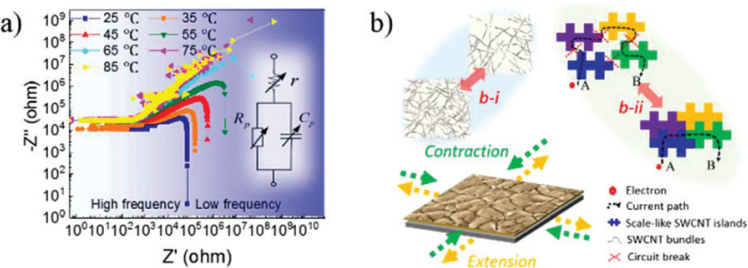

c)
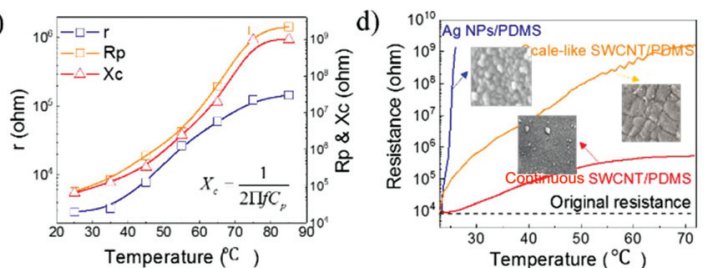

Fig. 4 Theoretical analysis of the sensing capability of SSPFs. (a) Electrochemical impedance spectroscopy of SSPF-2 at different temperatures; inset is a possible equivalent circuit generated via a MATLABbased impedance curve fitting program. (b) Illustration of the deformation of SSPFs induced via environmental stimuli, and the piezoresistive behavior of b-i: SWCNT bundles and b-ii: SSIs. (c) The resistance behavior in response to temperature of the components in the equivalent circuit $\left(r, R_{\mathrm{p}}, X_{\mathrm{c}}\right.$, respectively); note that $X_{\mathrm{c}}$ is the capacitive reactance from $C_{p}$, which is calculated via the inset equation. (d) The sensing performance of different nanomaterial/PDMS systems at different temperatures. The applied frequency scope in (a) and (c) is from $1 \mathrm{kHz}$ to $2 \mathrm{MHz}$.

The opening of the cracks in between the fragments can be regarded as the macroscopic piezoresistive behavior of the SSIs. Note that due to the release of the initial compressive stress, fragments can initially overlap in the relaxed state, as shown in Fig. S8. $\dagger$ This phenomenon can be confirmed via the change in roughness between WSPFs and SSPFs, as shown in Fig. S1. $\dagger$ When the SSIs become separated during the expansion of the film, the effective resistance of the junctions and then of the film increases, as demonstrated in Fig. $4 \mathrm{~b}$.

Stretching of the unscale-like fragments triggers the piezoresistive behavior of the SWCNT bundles, which can be regarded as the microscopic piezoresistive behavior of the SSIs (Fig. 4b). This piezoresistive behavior is typical in nanoparticles and can be attributed to the change in the resistance of the interparticle junctions when the coating is stretched. ${ }^{41}$

In practice, the two mechanisms have a synergetic effect, collectively contributing to the macroscopic change in resistance.

An equivalent circuit was generated via a MATLAB-based impedance curve fitting program (inset of Fig. 4a), which was composed of a series resistance $(r)$, a parallel resistance $\left(R_{\mathrm{p}}\right)$, and a capacitor $\left(C_{\mathrm{p}}\right)$.

To reveal the contribution of each mechanism to the individual components $\left(r, R_{\mathrm{p}}\right.$ and $\left.C_{\mathrm{p}}\right)$, the change of these components with temperature was summarized in Fig. $4 \mathrm{c} . R_{\mathrm{p}}$ and $X_{\mathrm{c}}\left(X_{\mathrm{c}}=1 /\left(2 \pi f C_{\mathrm{p}}\right)\right)$ increased up to $10^{9} \mathrm{ohm}$ above $75^{\circ} \mathrm{C}$, indicating that the electrical circuit was nearly completely open. Meanwhile, $r$ experiences only a relatively small change from $2.9 \mathrm{kohm}$ to $14 \mathrm{kohm}$, suggesting that the break in the circuit mainly resulted from the macroscopic piezoresistive behavior of SSIs (opening of the cracks between the fragments). Accordingly, we further confirm this deduction via experiments, comparing the resistance behaviors to the temperature of silver nanoparticles (Ag NPs)/PDMS, scale-like SWCNT/ PDMS and unscale-like SWCNT/PDMS films, as shown in Fig. 4d. Ag nanoparticles increased their resistance exponentially with minimal increases in temperature. This increase in resistance was likely due to the piezoresistive behavior of interparticle contacts (particle is around 2-10 $\mathrm{nm}$ ). Unscale-like SWCNT/PDMS films had a stable increase in resistivity with increasing temperature, likely as a result of the total microscopic piezoresistive behavior of SWCNTs. These results indicate that macroscopic piezoresistive behavior plays a more important role in resistance response than does microscopic piezoresistive behavior. Scale-like SWCNT/PDMS combines the benefits of both piezoresistive behaviors to achieve high sensitivity over a wide range of temperatures.

\section{Conclusions}

In conclusion, temperature-tunable microstructures (WSPFs and SSPFs) were fabricated and examined in detail. Based on their scale-like microstructure and the basic physical properties of each layer (low modulus and high CTE of PDMS, outstanding piezoresistive behavior of SWCNT), SSPFs demonstrated a superior sensing capability to environmental stimuli, such as pressure sensitivity up to $740.7 \mathrm{kPa}^{-1}$ and a thermal index up to $29.9 \times 10^{3} \mathrm{~K}$. These performances were also confirmed via a series of practical applications: accurate monitoring of the indicators of human health, weighing an insect (ant) and tracking its activities, gauging temperature, designing a thermal switch in a fire alarm system, and monitoring light. The performance of the sensor can be explained by the synergetic contribution of two piezoresistive mechanisms: a macroscopic mechanism associated with the crack opening and a microscopic mechanism associated with the change in distance between the CNTs inside the scale-like islands. Further investigations are necessary to explore additional sensing behaviors for SSPFs, including responses to humidity and light, and to investigate other applications of mechanical buckling, like superhydrophobic structures and nanogenerators.

\section{Experimental}

\section{Materials}

We purchased PDMS (Sylgard® 184) with a base and a curing agent from Dow Corning Inc. The PEDOT/PSS aqueous dispersion (1.3 wt\%, Clevios $\left.{ }^{\mathrm{TM}} \mathrm{PH} 1000\right)$ was purchased from $\mathrm{HC}$ Starck, Inc. Single-walled carbon nanotubes (SWCNTs) were purchased from Cheap Tubes, Inc. with an outer diameter of 1-2 $\mathrm{nm}$, a length of $5-30 \mu \mathrm{m}$, and over $95 \mathrm{wt} \%$ purity and $2.56 \mathrm{wt} \% \mathrm{COOH}$ groups. The SWCNT/PEDOT:PSS ink ( $1 \mathrm{mg} \mathrm{mL}{ }^{-1}$, SWCNT/PEDOT:PSS = 1/1 wt\%) and silver nanoink (10 wt\%) were self-prepared in the lab according to the 
methods we previously described. ${ }^{42}$ Typical SWCNT ink and silver ink can be seen in Fig. S9. $\uparrow 50 \mu \mathrm{m}$ PET films were purchased from Teonex® Inc., and Teflon film tape (thickness = 0.08-013 mm) was purchased from Shaheen Enterprises. Deionized (DI) water was used in all experimental processes.

\section{Preparation of nanomaterial/PDMS films}

For WSPFs, we dropped the prepared SWCNT ink onto a plasma-pretreated PDMS film (cured at $70{ }^{\circ} \mathrm{C}$ for $6 \mathrm{~h}$, thickness $=$ $200 \mu \mathrm{m}$, base/curing agent $=10: 1)$ using a Thermo Scientific Finnpipette $(0.2-2 \mu \mathrm{l})$ with a controlled concentration of $3 \mu \mathrm{cm}^{-2}$. Then, these films were dried in an oven at different temperatures $\left(50{ }^{\circ} \mathrm{C}\right.$ : WSPF-1, $100{ }^{\circ} \mathrm{C}$ : WSPF-2, $150{ }^{\circ} \mathrm{C}$ : WSPF-3, respectively). After $1 \mathrm{~h}$, the films were cooled to room temperature.

For SSPFs, the above-prepared WSPFs were stretched by hand along their $X$ - and $Y$-axes 10 times and then fully rubbed for $10 \mathrm{~s}$.

For the silver nanoparticle/PDMS film, the same dropping process was used. The film was then dried at room temperature for $3 \mathrm{~h}$.

Q4 For the patterned SWCNT/PDMS film, the SWCNT with " $S$ " configuration was fabricated on the PDMS film (thickness $=$ $200 \mu \mathrm{m}$ ) via a template, and then dried at room temperature for 3 hours.

\section{Fabrication of the pressure-sensing device}

SSPF-based pressure-sensing unit. First, the same plasmapretreated PDMS film used above was attached to a PET film with a hole drilled via a punch (diameter $=8 \mathrm{~mm}$ ). The SWCNT ink was then deposited into the hole at the same concentration by the same method. After drying, this PDMS film with a circular SWCNT pattern (diameter $=8 \mathrm{~mm}$ ) was peeled from the PET film.

Second, another PET film with a hole (diameter $=6 \mathrm{~mm}$ ) was prepared, and two silver electrodes were fabricated via the direct-writing method. Accordingly, a new PDMS film was attached to this patterned PET film via ethanol, dried for $30 \mathrm{~min}$ at $60{ }^{\circ} \mathrm{C}$, and then peeled off. The object of this method was to successfully transfer a nano-sized PDMS layer onto this patterned PET film.

Third, the patterned PDMS film was attached to the patterned PET film (pattern center to hole center) after plasma treatment of both films via ethanol and cured at $100{ }^{\circ} \mathrm{C}$ for $1 \mathrm{~h}$. Copper wires were bonded on the surface of the silver electrodes on PET using silver paste, which was then cured on a hot plate at $100^{\circ} \mathrm{C}$ for $1 \mathrm{~h}$.

Due to the excellent sealing performance of PDMS, both films bonded well for the successful transfer of the nano-sized PDMS layer. The SSPF-based pressure-sensing array was fabricated via the same methods described above. More details about the fabrication processes and the typical pressuresensing devices can be seen in Fig. S10.†

\section{Characterization and measurements}

The surface morphology of the prepared WSPFs and SSPFs was examined by scanning electron microscopy (SEM, Quanta 600,
FEI Company) and atomic force microscopy (AFM, Veeco, Dimension 3100). Sheet resistance was measured using a 4-point probe system (Pro4-440N, Lucas Labs), and the cyclic mechanical tests were performed on a PC-controlled universal test machine (Instron 5944 with a $5-\mathrm{N}$ load cell) with a PCrecordable multimeter (Agilent 34401A). Temperature was controlled on a hotplate (CB162, Stuart Company), and the temperature was controlled by using a PC-recordable thermometer (TC-08, Pico Tech.). The variation in the impedance of SSPF-2 at different temperatures was monitored by using a LCR meter (Agilent E4980A). Default conditions were set as ambient environmental conditions with a relative humidity $(\mathrm{RH})$ of $41.3 \%$ and a temperature of $24.3{ }^{\circ} \mathrm{C}$. Note that more details about setups of pressure/temperature sensing tests can be seen in Fig. S11 and S12. $\dagger$

We designed a fire alarm system to assess the potential of SSPFs as a thermal switch using a digital logic-based circuit, which was composed of a power supply ( $5 \mathrm{~V})$, a regular LED, a regular loudspeaker, a controller resistance $\left(R_{\mathrm{c}}\right)$, a NOT gate, and an inverter IC. More details can be seen in Fig. 3f.

As a working mechanism. (1) At low temperatures, the sensor provides a comparatively low resistive path and conducts the current, which passes through the controller resistor, $R_{\mathrm{c}}$, making a voltage divider circuit. As the resistance value of $R_{\mathrm{c}}$ is set very high compared to the sensor resistance $\left(R_{\mathrm{c}} \gg R_{\mathrm{S}}\right)$, almost the entire battery voltage $(V)$ appears at point $\mathrm{A}$, which is considered as the "Digital 1" by the NOT gate of the inverter, IC. Thus, the output of the NOT gate remains low (Digital 0), making it incapable of turning on the LED and initiating the alarm.

(2) At high temperatures, the sensor provides a large resistance compared to $R_{\mathrm{c}}\left(R_{\mathrm{S}} \gg R_{\mathrm{c}}\right)$ and hence a low voltage appears at point $\mathrm{A}$, which is considered as the Digital 0 by the NOT gate. In this situation, the NOT gate output becomes high (Digital 1), enabling it to switch on the LED and ring the alarm.

\section{Acknowledgements}

We express gratitude to the Baseline Funding from the King Abdullah University of Science and Technology (KAUST) for financial support. This work was also partially supported by the key discipline fund of Shanghai (B117).

\section{References}

1 Y. S. Kim, R. Tamate, A. M. Akimoto and R. Yoshida, Mater. Horiz., 2014, 4, 38.

2 K. Efimenko, M. Rackaitis, E. Manias, A. Vaziri, L. Mahadevan and J. Genzer, Nat. Mater., 2005, 4, 293.

3 D. H. Kim, J. H. Ahn, W. M. Choi, H. S. Kim, T. H. Kim, J. Song, Y. Y. Huang, Z. Liu, C. Lu and J. A. Rogers, Science, 2008, 320, 507.

4 F. Rickhey, M. Kim, H. Lee and N. Kim, Mater. Des., 2015, 65, 995. 
5 S. Huang, C. F. Guo, X. Zhang, W. Pan, X. Luo, C. Zhao and H. Wu, Small, 2015, 11, 5712.

6 T. Ohzono, H. Watanabe, R. Vendamme, C. Kamaga, T. Kunitake, T. Ishihara and M. Shimomura, Adv. Mater., 2007, 19, 3229.

7 S. Deng and V. Berry, Mater. Today, 2016, 19, 197.

8 M. Y. Li, M. Zu, J. S. Yu, H. F. Cheng and Q. W. Li, Small, 2017, DOI: 10.1002/smll.201602994.

9 W. H. Koo, S. M. Jeong, F. Araoka, K. Ishikawa, S. Nishimura, T. Toyooka and H. Takezoe, Nat. Photonics, 2010, 4, 222.

10 W. Xu and T. Lee, Mater. Horiz., 2016, 3, 186.

11 C. M. Stafford, C. Harrison, K. L. Beers, A. Karim, E. J. Amis, M. R. VanLandingham, H. C. Kim, W. Volksen, R. D. Miller and E. E. Simonyi, Nat. Mater., 2004, 3, 545.

12 S. J. Kim, H. R. Cho, K. W. Cho, S. Qiao, J. S. Rhim, M. Soh, T. Kim, M. K. Choi, C. Choi, I. Park and N. S. Hwang, ACS Nano, 2015, 9, 2677.

13 N. Bowden, S. Brittain, A. G. Evans, J. W. Hutchinson and G. M. Whitesides, Nature, 1998, 393, 146.

14 E. Cerda and L. Mahadevan, Phys. Rev. Lett., 2003, 90, 074302 .

15 Z. Huang, W. Hong and Z. Suo, Phys. Rev. E: Stat. Phys., Plasmas, Fluids, Relat. Interdiscip. Top., 2004, 70, 030601.

16 N. Bowden, W. T. Huck, K. E. Paul and G. M. Whitesides, Appl. Phys. Lett., 1999, 75, 2557.

17 D. Y. Khang, J. A. Rogers and H. H. Lee, Adv. Funct. Mater., 2009, 19, 1526.

18 Kenry, J. C. Yeo, J. Yu, M. Shang, K. P. Loh and C. T. Lim, Small, 2016, 12, 1593.

19 G. Kamita, B. Frka-Petesic, A. Allard, M. Dargaud, K. King, A. G. Dumanli and S. Vignolini, Adv. Opt. Mater., 2016, 4, 1950.

20 C. Yeom, K. Chen, D. Kiriya, Z. Yu, G. Cho and A. Javey, Adv. Mater., 2015, 27, 1561.

21 W. Zeng, I. Jacobi, D. J. Beck, S. Li and H. A. Stone, Lab Chip, 2015, 15, 1110.
22 S. Y. Hong, Y. H. Lee, H. Park, S. W. Jin, Y. R. Jeong, J. Yun, 1 I. You, G. Zi and J. S. Ha, Adv. Mater., 2016, 28, 930.

23 S. Choi, J. Park, W. Hyun, J. Kim, J. Kim, Y. B. Lee, C. Song, H. J. Hwang, J. H. Kim, T. Hyeon and D. H. Kim, ACS Nano, 2015, 9, 6626.

24 L. Kergoat, B. Piro, D. T. Simon, M.-C. Pham, V. Noël and M. Berggren, Adv. Mater., 2014, 26, 5658.

25 S. Jana, A. Zhamu, W. H. Zhong, Y. X. Gan and J. J. Stone, Mater. Manuf. Processes, 2007, 23, 102.

26 L. Pocivavsek, R. Dellsy, A. Kern, S. Johnson, B. Lin, K. Y. C. Lee and E. Cerda, Science, 2008, 320, 912.

27 Z. Pan, N. Liu, L. Fu and Z. Liu, J. Am. Chem. Soc., 2011, 133, 17578.

28 S. Yao and Y. Zhu, Adv. Mater., 2015, 27, 1480.

29 T. Yang, W. Wang, H. Zhang, X. Li, J. Shi, Y. He, Q. S. Zheng, Z. Li and H. Zhu, ACS Nano, 2015, 9, 10867.

30 Y. Tai, M. Mulle, I. A. Ventura and G. Lubineau, Nanoscale, 2015, 7, 14766.

31 L. Pan, A. Chortos, G. Yu, Y. Wang, S. Isaacson, R. Allen, Y. Shi, R. Dauskardt and Z. Bao, Nat. Commun., 2014, 5, 3002.

32 Y. L. Tai and Z. G. Yang, J. Mater. Chem. B, 2015, 3, 5436.

33 X. Wang, Y. Gu, Z. Xiong, Z. Cui and T. Zhang, Adv. Mater., 2014, 26, 1336.

34 B. Nie, R. Li, J. Cao, J. D. Brandt and T. Pan, Adv. Mater., 25 2015, 27, 6055.

35 Y. Tai and Z. G. Yang, Langmuir, 2015, 31, 13257.

36 Y. Tai, G. Lubineau and Z. Yang, Adv. Mater., 2016, 28, 4665.

37 Q. Li, C. Liu, Y. H. Lin, L. Liu, K. Jiang and S. Fan, ACS Nano, 2015, 9, 409.

38 K. M. F. Shahil and A. A. Balandin, Solid State Commun., 2012, 152, 1331.

39 A. Feteira, J. Am. Ceram. Soc., 2009, 92, 967.

40 C. Yan, J. Wang and P. S. Lee, ACS Nano, 2015, 9, 2130.

41 Y. Tai and G. Lubineau, Adv. Funct. Mater., 2016, 26, 4078.

42 Y. Tai, Y. X. Wang, Z. G. Yang and Z. Q. Chai, Surf. Interface Anal., 2011, 43, 1480. 